\title{
Battery Pot Plants with Magnets and Adjacent Balloon as Substitutes for Light
}

\author{
Lena J-T Strömberg ${ }^{1 *}$ \\ ${ }^{1}$ Department of Solid Mechanics, KTH Royal Institute of Technology, Stockholm, Sweden \\ Received 26 February 2021; Revised 14 May 2021; Accepted 23 May 2021; Published 01 June 2021
}

\begin{abstract}
In a future of clean technologies and innovations, it is important with knowledge of new Power sources in terms of the impact on living plants and surrounding life. Organic battery plants with electric components are constructed and analysed. The purpose is to study how illumination, i.e. light energy, can be replaced with electricity and matter. The novelty of the paper is the constructions, namely a balloon capacitor next to a current amplifier in the lines of a pot battery with a plant that should prosper, and the related analysis. Also, certain aspects of small magnetic fields alone are invoked.
\end{abstract}

Keywords: Flower Power; Pottery Cultivation; Organic Adaption; Evolution.

\section{Introduction}

The majority of vegetation uses sunlight and water moisture as inputs for growth. In addition to "green matter", the output is cleaner air and oxygen. In the present paper, species that usually benefit from traditional energy sources will be exposed to alternatives in terms of electricity, magnetic fields, reflecting matter, and adjacent structures. A search on related subjects gives commercial sites providing monitors, indicating electrodes are put on trees and used as sensors [1], and a review article by Teng et al. (2018) [2].

Teng et al. (2018) [2], appears generally inclusive and contains, e.g., results with galvanic voltages of $0.7 \mathrm{~V}$, but not this direction into developments for individual plants, e.g. on how to use induction and put in series as in Strömberg $(2020,2021)[3,4]$. The innovation of this article is to combine the pot plant batteries with components that enhance growth (under certain circumstances), control moisture, and magnify the electric power. Another feature covered is analysis, such that electricity and other interactions are understood in its formula language. Some new analysis with references quoted below, together with experiments, provided the basis for choosing the present arrangements, models, and conclusions. In Strömberg $(2020,2021)[3,4]$, organic battery plants in conjunction with electric components were studied; e.g., the capacity to produce electric power with amplifying Sun Catchers or magnetic induction. Two experiments with such materials are described.

- The response of plants to magnetic fields;

- A balloon, which provides energy due to its presence with low weight and since it moves an electric capacitor;

* Corresponding author: lena_str@hotmail.com

doi) http://dx.doi.org/10. 28991/HEF-2021-02-02-05

> This is an open access article under the CC-BY license (https://creativecommons.org/licenses/by/4.0/).

(C) Authors retain all copyrights. 


\section{Magnetic Fields Adjacent To Plants}

The pot in Figure 1 is magnetic iron, and the plant is placed under a magnet attached as a pendulum. Such arrangements appear to slow down the time for the plants, i.e., that they are captured in a static tranquillity with the magnetic field lines in a constant vertical direction. A small magnet, attached to the plant, interacts with the larger, such that the latter spins in oscillations around the vertical axis, if not too close such that it fastens. Hereby, an oscillating magnetic field acts close to the leaves.

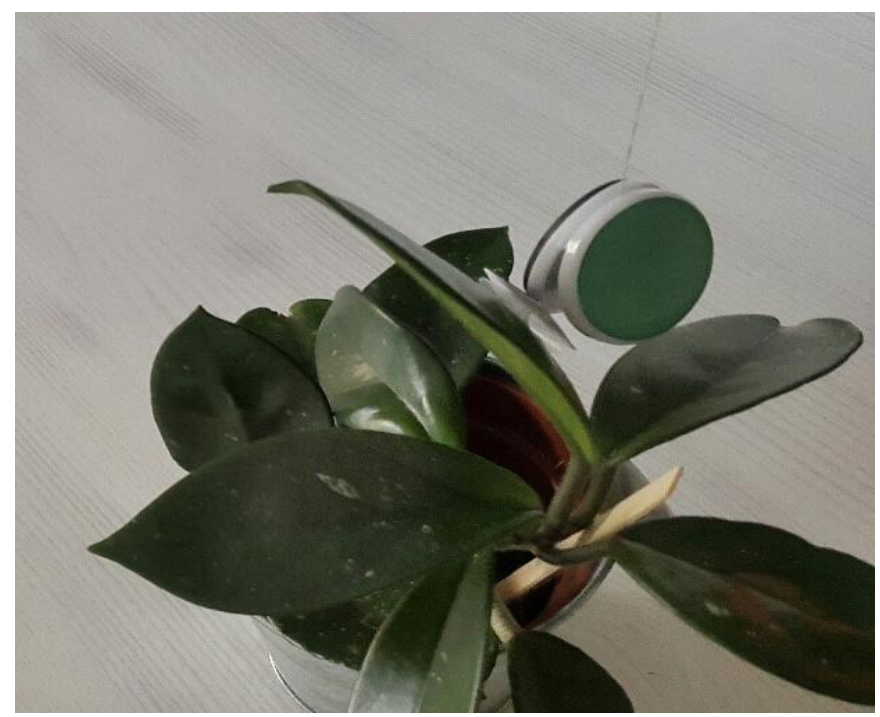

Figure 1. A plant in a magnetic pot, below a magnet attached in the roof as a pendulum

Hoya carnosa and several other species were found to be conserved and also grow in a ferromagnetic surrounding with sparse illumination. In Figure 2, a real flower is combined with magnetic induction when a small magnet is attached to its leaves. Possibly, a plant may learn to move the leaves in order to obtain electricity.

\section{A light Balloon in a Pottery Arrangement where Sparse Illumination}

\subsection{Balloon Field and Electricity as Substitute for Light and Water}

The plant arrangement subject to study is seen in Figure 2. A foil with lines on an elastic beam is a capacitor activated by mechanical motion, c.f. [4]. At the end, a large balloon is attached, such that motions in air layers are transmitted.

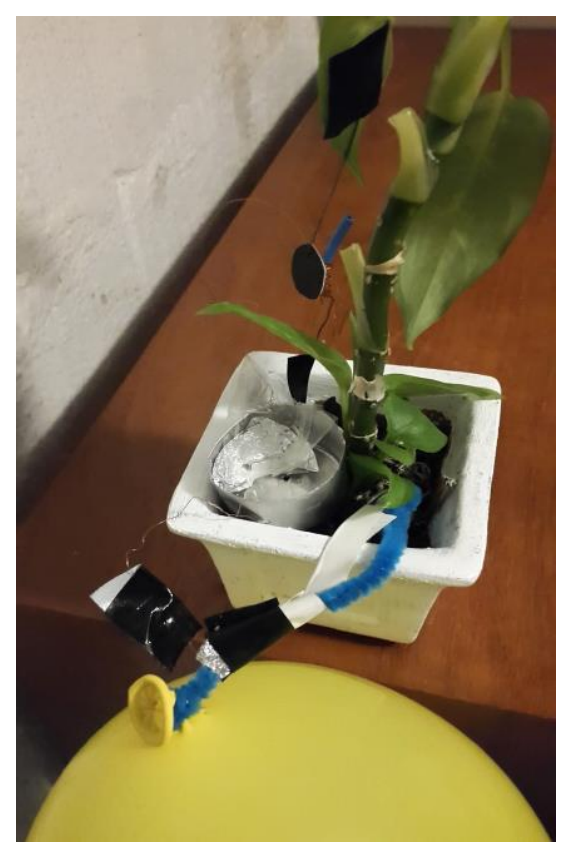

Figure 2. Dieffenbachia seguine, in pot with soil, electrodes, inductor coil behind a magnet (black), capacitor on the blue string and Sun Catcher Cup. When the balloon moves, the capacitor becomes active creating AC. The Cup collect extra energy from light and heat, if any. This pottery arrangement shows that a plant with access to electromagnetic energy (plausibly able to release beneficially,) can adjust and grow in a dark surrounding. 
The purpose of the balloon is two-folded. It moves easily at small indoor air-flows. It is light and spherical, hence it does not pin-point the gravity direction, which may influence the plant to not expect sun light from above. Instead, it may profit on the electromagnetic fields present in the electric lines.

The plant in Figure 2 was potted up in a battery without cup-amplification and capacitor, only electrodes and, at occasions; magnetic induction, before this experiment. Then, it was moved to a more dark environment with sparse artificial light less than $4 \mathrm{~h}$ a day and temperature $16^{\prime} \mathrm{C}$. First, some leaves in the center was lost. The roots were partly demolished so the small pot should remain sufficient. During a testing period of $14+28$ days, the plant continued to grow (first, especially the ground vegetation, such that a bit polarised). It probably got used to the electric energy instead of water and light.

\section{Results}

Primarily, Dieffenbachia seguine grows upwards and on the shadow side by bending towards the light. A part of spherical increase on different scales is present, especially for the ground vegetation on a meso/macro-level. The condition after 14 days indicates that the plant may use electric power as a complement to (part-time a substitute for) water and light. However, it is known that the loads travel less in dry soil, so it is possible that the presence of the balloon and/or magnetic induction is the source of energy during these times. The plant could also be at rest, not being powered by electricity and growing on its own stored energy.

\subsection{Model and Analysis}

Here, growth will be related to cohomologies with magic(n). Introductory, we will argue for that the presence of space and matter in terms of a balloon may substitute light with other energy, due to induced dimensions. Possibly Sunlight is somewhat spherically distributed, since it is remote. That is similar to an isotropic state, described as proportional to eye (3). The balloon plausibly induces air layers with a hydrostatic pressure $p=c$ eye(3), where $\mathrm{c}$ is a scalar. In that sense, the balloon could replace a Light source. Also the Solar Breeze function [3] may be replaced with pressure: at least theoretically: In Strömberg (2020) [5]; Section 4.1, solutions for a bounded area in a flow with pressure, were outlined, e.g. swimmers [6], in an oscillating pressure. A linearisation gives pointwise pressure oscillation, at a transient area, or an oscillating area when the pressure is transient or hyperbolic. This corresponds to moving and growing leaves.

Since the electro-magnetism in the lines is related to its motion, there is additional energy of the kind provided by light. Here, this is recognized:

- By the roots in terms of particle loads traveling between anode and cathode in the soil.

- As electric load due to a macro strain gauge from the elastic string attached to the balloon

- By induction when the small leave-magnet moves near the coil

Other related analysis are Rindler dust [7], swimmers and general composed flows [6,8]. The function of the foil in the capacitor can be understood in terms of reflected and refracted traveling waves by Chafin (2016) [9], where also acoustics are addressed. Many plants grow similar to adjacent and that is more often a law of nature and also ruling in agriculture. Next, the space with one plant and a balloon will be analysed in terms of cosmology, multi-dimensions and magic(n).

\subsection{Interpretation from Cosmology}

One way to characterise interaction with the balloon is gravity. Within cosmology, different kinds of modeling in multi-dimensions $\mathrm{n}$, give a gravity measure $\mathrm{Gn}$, proportional to gravity in a hyperspace [10]. Between 5 and 4 dimensional spaces, two are G5 $=\mathrm{G} 4 / \mathrm{k}$ where $\mathrm{k}$ is a curvature and $\mathrm{G} 5=\mathrm{G} 4 \times \mathrm{V}_{5-4}$ where $\mathrm{V}_{5-4}$ is a compact space volume for the (5-4) $)^{\text {th }}$ dimension. Hereby, gravity in the super-space increases with size of the extra dimension; e.g. a large balloon.

\subsection{The Matrix Magic(n)}

Magic(n) is pure number theory but since a decomposed structure in e.g. 3D is consistent with phenomena and processes in the Physical space, we will consider it a rule for reduction and subdivisions also when space becomes multidimensional due to adjacent matter and structure. In another project, decompositions were derived such that magic(3) were represented in two ways:

- With a pressure proportional to eye(3) and a subspace with two equal in size and opposite sign. (5eye(3) and +10)

- With the largest eigen-value in one direction, and two smaller, equal in size and opposite sign. (15 and +-5). 
If we assume that a pressure-state and a surface induce magic(3) in their first decomposition, the other space, with one larger eigen-value and another plane, may also exist in the surrounding. Together with stem and leaves, higher dimensions are introduced, and e.g. growth is when these add to fulfil magic(n).

\section{Conclusion}

EM arrangements for cultivation where sparse illumination were proposed and tested for two months. It was successful for these plants, however minor growth was triggered by the demolition of the roots with a knife. There were also species (both new small and older) that, after $\sim 3$ weeks, displayed more withering than growth. A conclusion made by observations of other plants with electrodes is that suitable moisture gradients, and possibly equivalents are created. This is beneficial for keeping moisture, and the plant seemed to learn to use the gradients by growth. There are limitations to this conclusion since there were moments when electricity had to be removed after initial breeding. The magnet in Figure 1 spins due to magnetic interaction, but if bouncing, this is more often dominant. Bouncing is also a d.o.f. close to a balloon, and is present in a potential for quantum cosmology [11]. Such motions provided by pressure in conjunction with leaves were sketched in Section 4. In modeling, we listed phenomenological aspects related to space curvature and matter, followed by qualitative and quantitative results in terms of magic(n).

\section{Declarations}

\subsection{Data Availability Statement}

Data sharing is not applicable to this article.

\subsection{Funding}

The author received no financial support for the research, authorship, and/or publication of this article.

\subsection{Institutional Review Board Statement}

Not applicable.

\subsection{Declaration of Competing Interest}

The author declare that there is no conflict of interests regarding the publication of this manuscript. In addition, the ethical issues, including plagiarism, informed consent, misconduct, data fabrication and/or falsification, double publication and/or submission, and redundancies have been completely observed by the author.

\section{References}

[1] ICT International, (2021). Available online: www.ictinternational.com/casestudies/forest-sensors-and-monitoring-technologies/ (accessed on June 2021).

[2] Teng, H. C., Kok, B. C., Uttraphan, C., \& Yee, M. H. (2018). A Review on Energy Harvesting Potential from Living Plants: Future Energy Resource. International Journal of Renewable Energy Research, 8(4), 2598-2614.

[3] Strömberg L. (2021). A prelude to a Sea Breeze, Methods in Modeling and Experiments; Lambert Academic Publishing, OmniScriptum, Saarbrücken, Germany.

[4] Strömberg L. (2021) Micro-Power-Harvesting of Electricity in Pot Plants with Components. Further Materials Science 1(1) 614.

[5] Strömberg, L. J.-T. (2020). Differentiations of Nonlinear Functions Related to States in Magic(n), Cosmology and the Poincare Conjecture. Journal of Human, Earth, and Future, 1(4), 197-201. doi:10.28991/hef-2020-01-04-05.

[6] Van Rees, W., Gazzola, M., \& Koumoutsakos, P. (2013). Optimal shapes for anguilliform swimmers at intermediate Reynolds numbers. Journal of Fluid Mechanics, 722, R3. doi:10.1017/jfm.2013.157.

[7] Rindler W. (1991). Introduction to special relativity, Oxford Science Publications, Clarendon Press; 2nd edition (July 11, 1991).

[8] Walther, J. H., \& Koumoutsakos, P. (2001). Three-Dimensional Vortex Methods for Particle-Laden Flows with Two-Way Coupling. Journal of Computational Physics, 167(1), 39-71. doi:10.1006/jcph.2000.6656.

[9] Chafin, C. E. (2016). Wave-Flow Interactions and Acoustic Streaming. Available online: https://arxiv.org/abs/1602.04893 (accessed on June 2021).

[10] Banerjee, S., Danielsson, U., \& Giri, S. (2021). Bubble needs strings. Journal of High Energy Physics, 2021(3), 1-18. doi:10.1007/jhep03(2021)250.

[11] Danielsson, U. H., Panizo, D., Tielemans, R., \& Van Riet, T. (2021). Higher-dimensional view on quantum cosmology. Physical Review D, 104(8), 86015. doi:10.1103/PhysRevD.104.086015. 